\title{
NONIMBEDDABLE NOETHER LATTICES
}

\author{
KENNETH P. BOGART
}

1. Introduction. The concept of a Noether lattice was introduced by R. P. Dilworth [4] as a generalization of the concept of the lattice of ideals of a Noetherian ring. A Noether lattice is a modular multiplicative lattice satisfying the ascending chain condition in which every element is a join of elements called principal elements. The principal elements are defined by a pair of identities satisfied by the principal ideals of a commutative ring.

It is possible to obtain complete descriptions of the structure of certain restricted classes of Noether lattices [1], [2], [3], [4]. In this paper we obtain another such description. A multiplicative lattice $L$ is said to have the trivial multiplication if

$$
\begin{array}{ll}
A I=A & \text { for all } A \in L \\
A B=0 & \text { for all } A, B \in L \text { such that } A, B \neq I .
\end{array}
$$

The main result of this paper is as follows.

$A$ lattice $L$ may be represented as a Nother lattice with the trivial multiplication if and only if $L$ is a finite-dimensional modular lattice in which every element except the unit element is a join of atoms.

This result will be used to prove the existence of Noether lattices which cannot be imbedded in the lattice of ideals of any Noetherian ring. The result given above will also be used to prove a generalization of the fact that if $M$ is a maximal (proper) ideal of a Noetherian ring $R$, then the ring $A / M A$ is a vector space over $R / M$ for all ideals $A$ of the ring. The generalization states that if $M$ is a maximal (proper) element of a Noether lattice, then the quotient sublattice $A / A M$ is a complemented modular lattice for all $A$ in $L$.

2. A review of basic concepts. A multiplicative lattice is a complete lattice provided with a commutative, associative, join-distributive multiplication for which the unit element is a multiplicative identity. We use juxtaposition to denote multiplication, $\vee$ and $\wedge$ to denote the lattice operations, and $\leqq$ to denote the lattice partial ordering. If $A$ and $B$ are elements of $L$, the residual of $A$ by $B, A: B$ is defined to be the join of all $Z \in L$ such that $Z B \leqq A$. In [2] it is shown that in modular lattices the definition of principal elements given in [4] may be restated as follows. If $L$ is a modular multiplicative lattice, then an element $E$ of $L$ is principal if and only if

Presented to the Society, April 19, 1969; received by the editors October 21, 1968. 


$$
A \wedge E=(A: E) E \quad \text { for all } A \in L
$$

and

$$
(A E): E=A \vee 0: E \quad \text { for all } A \in L .
$$

(It is a convention in multiplicative lattices that in an expression such as the right-hand side of 2.2 , the lattice operations are performed after the operations of multiplication or residuation.) The letter $E$ will be reserved for principal elements in this paper. Somewhat more complicated equations are used in [4] to define the concepts of meet-principal and join-principal, and an element is principal if it is both meet-principal and join-principal.

A Noether lattice is said to be local if it has precisely one maximal element, and in [1] it is shown that the principal elements in a local Noether lattice are precisely the join-irreducible elements.

In $\$ 5$ of this paper we will use the concept of a Noether lattice imbedding. A Noether lattice imbedding of a Noether lattice $L$ in a Noether lattice $L^{\prime}$ is defined in [1] as an isomorphism $\phi$ of $L$ into $L^{\prime}$ such that the images under $\phi$ of prime, primary, and principal elements in $L$ are prime, primary, and principal, respectively, in $L^{\prime}$. Thus, loosely speaking, $L$ can be imbedded in $L^{\prime}$ in such a way that the structure of $L$ as a Noether lattice is inherited from $L^{\prime}$.

\section{The basic theorem.}

TheOREM. 3.1. A lattice L may be represented as a Noether lattice with the trivial multiplication if and only if $L$ is a finite-dimensional modular lattice in which every element but $I$ is a join of atoms.

Proof. Suppose that $L$ is a Noether lattice with the trivial multiplication. Note first that $L$ is local, for if $I=A \vee B$ and $A, B \neq I$, then $I X=A X \vee B X=0$ for all $X \neq I$ in $L$. Thus $A=0, B=0$ and $I=0$. Now let $E \neq I$ be principal in $L$. Then (using Equation 2.1) if $X<E$, $X=(X: E) E=0$ since $(X: E) \neq I$. Thus the proper nonzero principal elements of $L$ are atoms and every element of $L$ but $I$ is a join of atoms. Let $M$ be the proper maximal element of $L$. Since $M^{2}=0$, $L=L / M^{2}$. By Theorem 6.2 of [2] $L / M^{2}$ is finite-dimensional. Therefore $L$ is finite-dimensional.

Now suppose that $L$ is a finite-dimensional modular lattice in which every element but $I$ is a join of atoms. Since $I$ is join-irreducible, the trivial multiplication on $L$ is a commutative, associative, join-distributive multiplication. To show that $L$ is a Noether lattice, we shall show that the atoms of $L$ are principal. In fact every element of a lattice with the trivial multiplication is join-principal. Since the 
multiplication is trivial, it is clear that

$$
\begin{array}{ll}
X: Y=M & \text { if } Y \leqq X \\
X: Y=I & \text { if } Y \leqq X
\end{array}
$$

for all $X$ and $Y$ in $L$, where $M$ is the proper maximal element of $L$. Now let $A$ and $C$ be elements of $L$. Then

$$
(A \vee I C): C=(A \vee C): C=I=A: C \vee I
$$

If $B \neq I$, then

$$
(A \vee B C): C=A: C=A: C \vee B,
$$

since $A: C=I$ or $A: C=M \geqq B$. Thus $C$ is join-principal and with $A=0$ and $C=E$, Equation (2.2) is satisfied. Now let $E$ be an atom of $L$. If $A \geq E$, then $A: E=M$. Therefore

$$
(A: E) E=M E=0=A \wedge E
$$

since $A \wedge E$ is zero because $E$ is an atom and $E \$ A$. If $A \geqq E, A: E=I$ and

$$
(A: E) E=I E=E=A \wedge E
$$

because $E \leqq A$. Thus $E$ satisfies (2.1) and is therefore principal. Since the atoms of $L$ are principal and every element of $L$ but $I$ is a join of atoms, and since $I$ and 0 are trivially principal, $L$ is a Noether lattice.

4. Example. We shall now construct a lattice $L$ which cannot be imbedded in the lattice of ideals of any ring. The elements of $L$ are the symbol $I$ and the elements of the lattice of subspaces of a nonDesarguesian projective plane. The partial ordering in $L$ is given by $I>X$ for all $X \neq I$ and by the partial ordering of the lattice of subspaces of the plane. Let the maximum element of the lattice of subspaces of the plane be $M$. Then $M / 0$ is a finite-dimensional complemented modular lattice, so that every element of $L$ but $I$ is a join of atoms and by Theorem 5.1, $L$ may be regarded as a Noether lattice with the trivial multiplication.

Now suppose that $\phi$ is a Noether lattice imbedding of $L$ in $L_{R}$, the lattice of ideals of a Noetherian ring $R$. Since $M$ is prime and each $X \leqq M$ is $M$-primary, the fact that $\phi$ is a Noether lattice imbedding implies that $\phi(M)$ is prime and $\phi(X)$ is $\phi(M)$-primary for all $X \leqq M$ in $L$. Because of this, we may assume without loss of generality that $R=[R / \phi(0)]_{(\phi(M))}$. Thus $L_{R}$ may be assumed to have the trivial multiplication. But for any Noetherian ring $R$ with maximal ideal $P, P / P^{2}$ is a vector space over the field $R / P$. This means that $\phi(M) / 0$ is a vector space. But the lattice of subspaces of a vector space is 
Arguesian since the lattice of subgroups of an Abelian group is Arguesian [6], so that the lattice of ideals of $R$ has no non-Arguesian sublattices. But $M / 0$ is non-Arguesian [6] so that $L$ cannot be imbedded in $L_{R}$.

5. An application. A basic theorem about Noetherian rings states that if $M$ is a maximal ideal of $R$ and $A$ is an ideal of $R$, then the quotient ring $A / A M$ is a vector space over $R / M$. Thus the ideals of $R$ between $A$ and $A M$ form a complemented modular lattice. If $R$ is local, then the number of elements in a minimal generating set for $A$ is the vector space dimension of $A / A M$. The next two results generalize these statements. Corollary 5.1 has also been proved by E. W. Johnson [5, Theorem 1.11].

Theorem 5.1. Let $L$ be a Noether lattice, and let $M$ be a (proper) maximal element of $L$. Then the quotient sublattice $A / A M$ is a finitedimensional complemented modular lattice.

Proof. We shall show that the set $L^{\prime}$ consisting of $I$ and the elements of $A / A M$ forms a Noether lattice with the trivial multiplication. Then Theorem 3.1 implies that $A / A M$ is a finite-dimensional complemented modular lattice.

Since $A / A M$ is a sublattice of $L, L^{\prime}$ is a sublattice $L$. To make $L^{\prime}$ a multiplicative lattice define $X \cdot Y=X Y \bigvee A M$ for all $X, Y$ in $L^{\prime}$. It is easy to see that with the multiplication $\cdot L^{\prime}$ is a multiplicative lattice. Thus there is a residuation in $L^{\prime}$ which we will denote by $\div$. If $X, Y \in L^{\prime}$ and $Y \$ X$,

$$
\begin{aligned}
X \div Y & =\vee\left\{Z \in L^{\prime} \mid Z \cdot Y \leqq X\right\} \\
& =\vee\left\{Z \in L^{\prime} \mid Z Y \vee A M \leqq X\right\} \\
& =\vee\{Z \leqq A \mid Z Y \leqq X\} \\
& =\vee\{Z \mid Z \leqq A, Z \leqq X: Y\} \\
& =A \wedge X: Y .
\end{aligned}
$$

Thus $X \div Y=A \wedge X: Y$ unless $Y \leqq X$, in which case $X \div Y=I$.

To show that $L^{\prime}$ is a Noether lattice, it is sufficient to show that the elements $E \vee A M$ are principal in $L^{\prime}$ for all principal elements $E \leqq A$ in $L$. In the proof of Theorem 3.1 it was shown that all elements of a lattice with the trivial multiplication are join principal, so that it is sufficient to show that the elements $E^{\prime}=E \bigvee A M$ satisfy equation 2.1 in $L^{\prime}$. If $E^{\prime} \leqq X, X \div E^{\prime}=I$ and

$$
E^{\prime}=X \wedge E^{\prime}=I E^{\prime}=\left(X \div E^{\prime}\right) E^{\prime} .
$$


If $E^{\prime} \$ X$, apply the definitions of · and $\div$ and (3.1) of [4] to get

$$
\begin{aligned}
\left(X \div E^{\prime}\right) \cdot E^{\prime} & =[X:(E \vee A M) \wedge A](E \bigvee A M) \wedge A M \\
& =(X: E \wedge A) E \bigvee A M \\
& =(X \wedge A E) \vee A M=A M,
\end{aligned}
$$

since $A E \leqq A M \leqq X$. But also, by modularity,

$$
\begin{aligned}
X \wedge E^{\prime} & =[X \wedge(A M \vee E)]=A M \vee(X \wedge E) \\
& =A M \vee(X: E) E,
\end{aligned}
$$

and since $X: E \neq I,(X: E) E \leqq A M$ and $X \wedge E^{\prime}=A M$. Thus the elements $A M \bigvee E$ are principal in $L^{\prime}$ for all principal elements $E \leqq A$ in $L$, and $L^{\prime}$ is a Noether lattice. This proves the theorem.

Corollary. 5.1. If $L$ is a local Noether lattice, then the number of elements in a minimal representation of $A$ as a join of principal elements is the lattice dimension of $A / A M$.

Proof. Let $A=E_{1} \bigvee \cdots \bigvee E_{n}$ be a minimal representation of $A$. If $E_{i} \leqq A M$ for some $E_{i}$, we can assume by renumbering that $E_{1}$ $\leqq A M$. Then using (3.2) of [4]

$$
\begin{aligned}
I & =A M: E_{1}=\left(E_{1} M \vee \cdots \vee E_{n} M\right): E_{1} \\
& =M \vee\left(E_{2} M \vee \cdots \vee E_{n} M\right): E_{1}
\end{aligned}
$$

which implies that $\left(E_{2} M \bigvee \cdots \vee E_{n} M\right): E_{1}=I$, so that $E_{1} \leqq E_{2}$ $\vee \cdots \vee E_{n}$ contradicting the minimality of the representation of $A$. Thus $A=\left(E_{1} \bigvee A M\right) \bigvee \cdots \vee\left(E_{n} \vee A M\right)$ is a minimal representation of $A$ as a join of principal elements in $A / A M$, so that by the proof of Theorem 3.1, the elements $E_{i} \bigvee A M$ are atoms. Since $A / A M$ is modular and since a minimal representation of $A$ as a join of atoms in $A / A M$ uses $n$ atoms, the lattice dimension of $A / A M$ is $n$.

\section{REFERENCES}

1. K. P. Bogart, Structure theorems for regular local Noether lattices, Michigan Math. J. 15 (1968), 167-176.

2. —, Distributive local Noether lattices (to appear).

3. - Idempotent Noether lattices (to appear).

4. R. P. Dilworth, Abstract commutative ideal theory, Pacific J. Math. 12 (1962), 481-498.

5. E. W. Johnson, A-transforms of Noether lattices, Dissertation, University of California, Riverside, 1966.

6. B. Jonsson, Modular lattices and Desargue's theorem, Math. Scand. 2 (1954), 295-314.

Dartmouth College 